\title{
Genetic evaluation of Cryptomeria japonica breeding materials for male-sterile trees
}

\author{
Takumi Tadama', Satoko Totsuka², Junji Iwai ${ }^{2}$, Kentaro Uchiyama³, Yoichi Hasegawa', Yoshinari \\ Moriguchi $^{1 *}$
}

\begin{abstract}
${ }^{1}$ Graduate School of Science and Technology, Niigata University, 8050, Ikarashi 2-Nocho, Nishi-ku, Niigata 950-2181, Japan
${ }^{2}$ Niigata Prefectural Forest Research Institute, 2249-5 Unotoro, Murakami, Niigata 958-0264, Japan

${ }^{3}$ Department of Forest Molecular Genetics and Biotechnology, Forestry and Forest Products Research Institute, 1 Matsu nosato, Tsukuba, Ibaraki 305-8687, Japan
\end{abstract}

*Corresponding author: Yoshinari Moriguchi, Email: chimori@agr.niigata-u.ac.jp

\begin{abstract}
Pyramiding of male-sterile genes in Cryptomeria japonica is currently being carried out in Niigata prefecture, Japan. This is the first attempt to apply pyramid breeding to forest trees. As the breeding materials for male sterility are limited, special attention must be given to increased genetic relatedness in the process of pyramid breeding to avoid the effects of inbreeding depression as much as possible. In this study, we estimated genetic relatedness based on 246 genome-wide SNP markers for male-sterile individuals in Niigata Prefecture $(n=6)$ and individuals doubly heterozygous for two male-sterile genes (hereafter referred to as "double-hetero") produced by markerassisted selection $(n=124)$. The pairwise relatedness estimates between male-sterile individuals selected from the same area in Niigata Prefecture were low $(-0.01 \pm 0.08$, mean \pm standard deviation), suggesting that there will be almost no negative effects even if the $F_{1}$ of these male-sterile individuals is used for artificial crossing. On the other hand, the pairwise relatedness between double-hetero individuals in this study was higher than the theoretical relatedness values, as individuals with the slightly higher relatedness were used as parents in artificial crossings. However, there was a large variance in pairwise relatedness for double-hetero individuals. This result suggested that it may be possible to avoid the adverse effects of inbreeding depression by using a pair of double-heteros with lower relatedness for artificial crossing, when we produce a double-homo using the limited breeding materials of malesterile individuals. It will also be important to continue additional selection of new breeding material for male sterility.
\end{abstract}

Keywords: Male sterility, Relatedness, Conifers, Marker-assisted selection (MAS), Pyramid breeding

\section{Introduction}

Cryptomeria japonica D. Don is an allogamous, monoecious, and wind-pollinated coniferous species. It is one of the major afforestation species in Japan, covering an area of 4.5 million hectares and accounting for approximately $44 \%$ of all artificial forests (Forestry Agency, 2014). The increased area of artificial forests of $C$. japonica has resulted in widespread $C$. japonica pollinosis, which is one of the most serious allergic diseases in Japan. Pollinosis caused by C. japonica pollen was first reported in 1964 (Horiguchi and Saito, 1964). The number of cases of $C$. japonica pollinosis has increased year by year, and $26.5 \%$ of the Japanese population is now allergic to $C$. japonica pollen (Baba and Nakae, 2008). The main countermeasures against $C$. japonica pollinosis are the use of individuals with low pollen (Iwasawa and Kodaira, 1995; Kondo, 2009) and/or male sterility (Taira et al., 2005). For individuals with low pollen, growth is superior as a result of the selection from plus trees. However, the amount of male strobili is not a stable trait, as some individuals with small amounts of strobili at a young age have a larger amount of male strobili as they age (Taira, 2004). In addition, because pollen contamination from external sources is a problem in most of the seed orchards that produce seeds for afforestation (Moriguchi et al., 2005a, b), there is a possibility that seeds with larger amounts of pollen than expected will be produced. On the other hand, male-sterile individuals, which 
were first discovered in Toyama Prefecture in 1992 (Taira et al., 1993), are considered an effective countermeasure against $C$. japonica pollinosis. To date, 23 individuals have been selected from seven prefectures (Saito, 2010). As a result of artificial crossings of various combinations, four male sterility genes, MS1, MS2, MS3, and MS4, have been identified, and it has been revealed that male-sterile individuals of $C$. japonica are dominated by a single recessive gene, the male-sterile gene (Taira et al., 1999; Yoshii and Taira, 2007; Miyajima et al., 2010; Saito, 2010). Currently, Niigata Prefecture, which has the largest number of breeding materials for male sterility in Japan, has six male-sterile individuals: Shindai-1, MS2 (Yoshii and Taira, 2007); Shindai-3, MS1 (Ueuma et al., 2009; Saito, 2010); Shindai-5, MS3 (Yoshii and Taira, 2007); Shindai-8, MS4 (Miyajima et al., 2010); Shindai-11, unknown (Miura et al., 2011); and Shindai-12, unknown (Miura et al., 2011). To carry out efficient tree breeding and seedling production using these limited breeding materials, it is necessary to use individuals with low relatedness for artificial crossing to avoid inbreeding depression (Taira et al., 2005). However, there is a possibility of kinship among male-sterile individuals in Niigata Prefecture because they were selected from artificial forests in the same geographical area.

In the process of producing seedlings of male-sterile individuals, low productivity and the amount of labor required are the primary issues (Saito, 2010). The expected proportion of male-sterile individuals among all seedlings is only $25 \%$ in crossings between individuals that are heterozygous for a male-sterile gene, and only $50 \%$ in crossings between a malesterile individual and an individual heterozygous for a malesterile gene. To theoretically increase the production efficiency of male-sterile seedlings up to $75 \%$, Moriguchi et al. (2017) suggested a method of selecting individuals with multiple male-sterile genes, i.e., a doubly homozygous tree such as $m s 1 m s 1 m s 2 m s 2$ (hereafter referred to as "double-homo"), and a doubly heterozygous tree such as Ms1ms1Ms2ms2 (hereafter referred to as "double-hetero"), using markers closely linked to male-sterile loci. Currently, to produce double-homo individuals experimentally according to Moriguchi et al. (2017), double-hetero individuals are selected as parents in artificial crossings performed in Niigata Prefecture. This is the first case of pyramid breeding in forest trees. The breeding materials that have the male-sterile gene, however, are limited. Kurinobu et al. (1991) reported the effects of inbreeding depression on seedling height in C. japonica 2-year-old seedlings (19-monthold seedlings). They found that the height of seedlings obtained by artificial crossings between half-siblings and between full-siblings were approximately $92 \%$ and $85 \%$, respectively, of the heights of seedlings obtained by artificial crossings between individuals with no relationship. Akashi (1994) reported that the height and basal diameter of 3-yearold seedlings obtained from artificial crossings between halfsiblings and between full-siblings were smaller than those from artificial crossings between unrelated individuals in $C$. japonica. In his study, the seedlings obtained from artificial crossings between full-siblings were the smallest. Therefore, we have to pay special attention to increased genetic relatedness in the process of pyramid breeding.

The objectives of this study were to evaluate the genetic relatedness of two populations: male-sterile individuals in Niigata Prefecture and double-hetero individuals selected using the method of Moriguchi et al. (2017). Our results will facilitate a model study of pyramid breeding in forest trees.

\section{Materials and Methods}

\section{Plant material}

To estimate pairwise relatedness between male-sterile individuals in Niigata Prefecture, we used not only six malesterile individuals selected from the Irihirose area of Niigata Prefecture (Shindai- $1,-3,-5,-8,-11$, and -12), but also three male-sterile individuals selected from the Nishi-Aizu area of Fukushima Prefecture (Fukushima-1, -2, and -3) (Fig. 1). The Nishi-Aizu area is close to the Niigata Prefecture. To estimate pairwise relatedness between double-hetero individuals, we used 124 double-hetero individuals that were produced by artificial crossing of two male-sterile individuals (Shindai- 1 and -3) and six breeding materials (i.e., two plus trees [Nakakubiki-4 and Santou-5], three individuals found to be tolerant to attack from Semanotus japonicus [Kamikiri Niigata-31, -38, and -47], and one individual found to be tolerant to snow pressure [Setsugai Niigata-20]) (Supplemental Table 1). In addition to these individuals, we added 19 breeding materials (i.e., 13 plus trees, five individuals tolerant to attack from Semanotus japonicus, and one individual tolerant to snow pressure) to estimate the pairwise relatedness (Fig. 1). Six of these were used to produce double-hetero individuals, as previously mentioned.

\section{DNA extraction and genotyping}

Total DNA was extracted from the needle leaves using the modified CTAB method (Tsumura et al., 1995). Single nucleotide polymorphism (SNP) genotyping was performed using a 48.48 Dynamic Array (Fluidigm, South San Francisco, CA, USA). A total of $6.25 \mathrm{ng}$ of genomic DNA per sample (at a concentration of $5 \mathrm{ng} / \mu \mathrm{L}$ ) was used for specific target amplification. The assays were carried out according to the manufacturer's instructions. The data obtained were analyzed using the Fluidigm SNP Genotyping Analysis software. To estimate the degree of genetic relatedness between each pair of individuals, we used a set of 246 high-resolution SNP markers selected by Uchiyama and Matsumoto (2018), which were widely selected across the C. japonica genome (Uchiyama et al., 2014) (Supplemental Table 2).

\section{Data analysis}

Pairwise genetic relatedness was calculated using COANCESTRY version 1.0.1.8 software (Wang, 2011). Queller and Goodnight's estimator (Queller and Goodnight, 1989) was calculated between all possible pairs of individuals using a file of known 


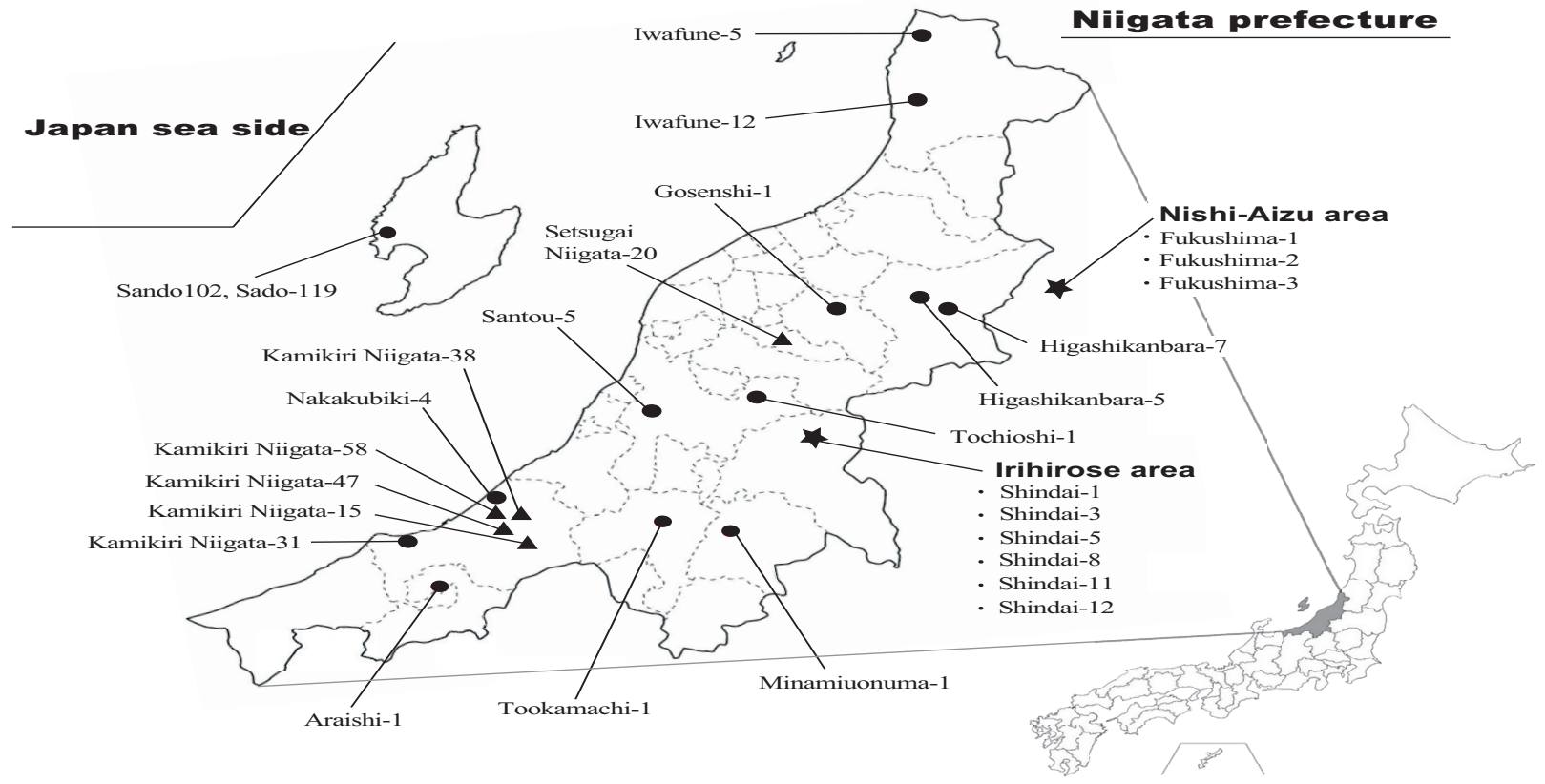

Figure 1

Locations of selected Cryptomeria japonica breeding materials used in this study ( $\star$ : male-sterile individuals, $\bullet$ :plus trees,

$\underline{\Delta}$ :individuals found to be tolerant to the attack from Semanotus japonicus, and an individual tolerant to the snow pressure in $C$. japonica). All trees were breeding materials in Niigata Prefecture, except three trees selected from Nishi-Aizu area in Fukushima Prefecture.

allelic frequencies of 257 individuals collected from natural populations of $C$. japonica (Supplemental Table 3).

Differences between theoretical and estimated relatedness values were determined using the Wilcoxon signed rank test. These analyses were performed using $\mathrm{R}$ ver. 3.4.1 (The R Foundation for Statistical Computing, 2017).

\section{Results and Discussion}

Pairwise relatedness between male-sterile individuals

The genotype data for male-sterile individuals was shown in Supplemental Table 4. The highest pairwise relatedness (0.18) was observed between Shindai-1 and Shindai-3 (Table 1). The average pairwise relatedness of all 36 pairs was $0.00 \pm 0.07$, and 32 pairs showed a relatedness of less than 0.1 . For male-sterile individuals selected from the Irihirose and Nishi-Aizu areas, the average pairwise relatedness was $-0.01 \pm 0.08$ and $0.02 \pm 0.03$, respectively, and they were also very low. Our results revealed that the relatedness between male-sterile individuals selected from the same area in Niigata Prefecture was low; therefore, there will be almost no negative effects even if the $F_{1}$ offspring from these male-sterile individuals are used for artificial crossings. Although the pairwise relatedness between Shindai-1 (ms2/ms2) and Shindai-3 (ms1/ms1) was a bit higher compared to the other pairs between male-sterile trees, artificial-crossing between them is not performed in conventional breeding because it can't produce male-sterile seedlings.

\section{Pairwise relatedness between double-hetero individuals}

The genotype data for double-hetero individuals and other breeding materials was shown in Supplemental Table 4. Theoretical relatedness values of 0 indicate unrelated pairs, values of 0.5 indicate full-siblings and/or parent-offspring pairs and values of 0.25 indicate grandparents-grandchildren pairs. The numbers of pairs for which the theoretical relatedness values were $0.5,0.375,0.3125,0.25,0.1875$, $0.125,0$ were $1,246,1,184,2,360,171,2,491,345$, and 2,356 pairs, respectively (Supplemental Table 5). Their average estimated relatedness values were $0.52 \pm 0.09,0.40 \pm 0.09$, $0.38 \pm 0.08,0.22 \pm 0.08,0.24 \pm 0.08,0.16 \pm 0.07$, and $0.01 \pm$ 0.07 , respectively, and all differed significantly from their theoretical relatedness values (Fig. $2, P<0.01$ ). Except for the pairs for which the theoretical relatedness value was 0.25 , the mean relatedness values estimated in this study were slightly higher than the theoretical values. These results may be due to the slightly higher relatedness between Shindai-1 and Shindai-3 $(0.18)$, because members of the $F_{1}$ of Shindai- 1 or Shindai-3 were used for all matings (Supplemental Table 1). This is supported by the result that the pairwise relatedness estimates in this study were not higher than the theoretical relatedness values in the pairs that did not use individuals derived from Shindai- 1 and Shindai-3 as parents. On the other hand, the variance in the pairwise relatedness 
Table 1

Pairwise relatedness coefficients using Queller and Goodnight's estimator (Queller and Goodnight, 1989) as implemented in COANCESTRY version 1.0.1.2 software (Wang, 2011) based on the 246 SNP markers.
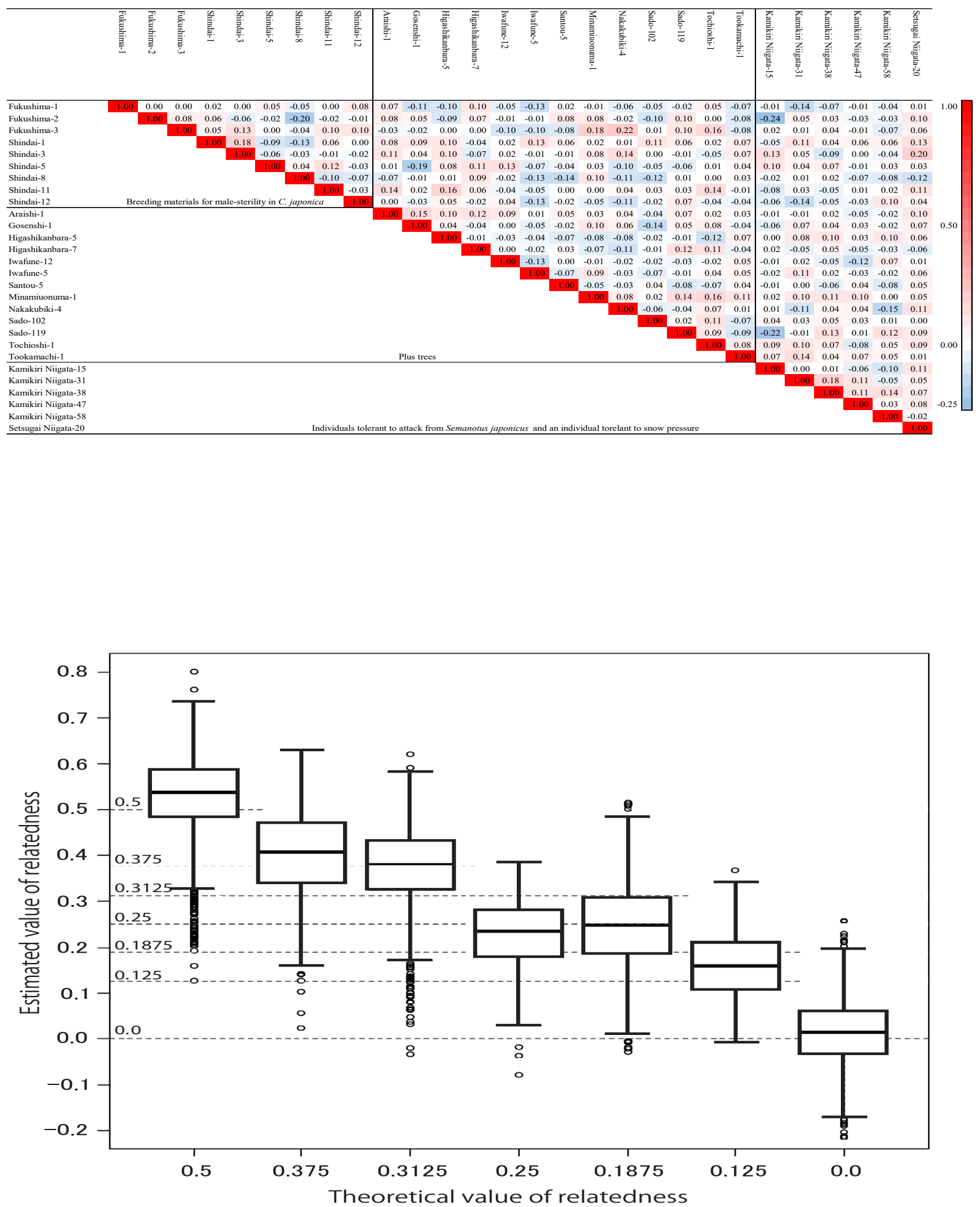

Figure 2

Relationships between the theoretical and estimated values of relatedness obtained from pairwise relatedness analyses between double-heterozygous individuals and 19 breeding materials (i.e., 13 plus trees, five individuals tolerant to attack from Semanotus japonicus, and one individual tolerant to snow pressure). 
estimates in this study was large (Fig. 2). Relatedness was calculated based on the proportion of alleles shared between individuals by homologous inheritance (Blouin, 2003). The average relatedness between parents and children and between full-siblings is 0.5 , but the patterns of shared alleles differ between these pairings. Between parents and children, the child always inherits $50 \%$ of an allele from each parent, but the variation in pairwise relatedness increases, because rates of shared alleles differ between full-siblings (Weir et al., 2006). Therefore, to produce a double-homo using the limited breeding materials of male-sterile individuals, it may be possible to avoid the adverse effects of inbreeding depression by using a pair of double-heteros with lower relatedness for artificial crossing. In the future, the selection of parents with lower relatedness for artificial crossings and the additional selection of new breeding materials for male sterility will be necessary to accelerate practical pyramid breeding.

\section{Acknowledgments}

The authors would like to thank Ms. Koshiba and Mr. Komatsu for their assistance with laboratory work. We also thank the Fukushima Prefectural Forest Experiment Stations for providing breeding materials for this study. This work was partly supported by the Science and Technology Research Promotion Program for Agriculture, Forestry, Fisheries, and Food Industry, and the NARO Bio-oriented Technology Research Advancement Institution (research program on the development of innovative technology).

\section{References}

Akashi T (1994) The selection effectiveness and inbreeding depression in the second generation of plus-trees of Cryptomeria japonica D. Don. Bulletin of the Forestry and Forest Products Research Institute 368:1-22 (in Japanese).

Baba K, Nakae K (2008) The national epidemiological survey of allergic rhinitis in 2008-comparison between 1998 and 2008. Progress in Medicine 28:20012012 (in Japanese).

Blouin MS (2003) DNA-based methods for pedigree reconstruction and kinship analysis in natural populations. Trends in Ecology and Evolution 18:503511. https://doi.org/10.1016/S0169-5347(03)00225-8

Horiguchi S, Saito Y (1964) Japanese cedar pollinosis in Nikko, Japan. The Japanese Society of Allergology 13:16-18 (in Japanese with English summary). https://doi.org/10.15036/arerugi.13.16

Forest Agency (2014) Statistical handbook of forest and forestry. Forestry Agen cy, Ministry of Agriculture, Forestry and Fisheries, Japan. P8-P9 (in Japanese).

Iwasawa M, Kodaira T (1995) Selection of Sugi (Cryptomeria japonica) producing few pollen in Chiba Pref. Transactions of the Meeting in Kanto Branch of the Japanese Forestry Society 47:55-56 (in Japanese).

Kurinobu S, Ohya K, Kawasaki H (1991) Inbreeding depression in two year old F2 seedling heights of sugi (Cryptomeria japonica) resulting from Full-sib and Half-sib matings. Journal of the Japanese Forestry Society 73:388-392. https://doi.org/10.11519/jifs1953.73.5 388

Kondo T (2009) Result of forest tree breeding (4) individuals for countermeasures against $C$. japonica pollinosis. Forest Tree Breeding 232:61-63 (in Japanese).
Miura S, Nameta M, Yamamoto T, Igarashi M, Taira H (2011) Mechanisms of male sterility in four Cryptomeria japonica individuals with obvious visible abnormality at the tetrad stage. Journal of the Japanese Forestry Society 93:1-7 (in Japanese with English summary). https://doi.org/10.4005/jjfs.93.1

Miyajima D, Yoshii E, Hosoo Y, Taira H (2010) Cytological and genetic studies on male sterility in Cryptomeria japonica D. Don (Shindai 8). Journal of the Japanese Forestry Society 92:106-109 (in Japanese with English summary). https://doi.org/10.4005/jijs.92.106

Moriguchi Y, Goto S, Takahashi M (2005a) Genetic management of seed orchards based on information revealed by molecular markers. Journal of the Japanese Forestry Society 87:161-169 (in Japanese with English summary). https://doi.org/10.4005/jifs.87.161

Moriguchi Y, Tani N, Itoo S, Kanechira F, Tanaka K, Yomogida H, Taira H, Tsumura Y (2005b) Gene flow and mating system in five Cryptomeria japonica D. Don seed orchards as revealed by analysis of microsatellite markers. Tree Genetics and Genomes 1:174-183. https://doi.org/10.1007/s11295-005-0023-z

Moriguchi Y, Totsuka S, Iwai J, Matsumoto A, Ueno S, Tsumura Y (2017) Pyramiding of male-sterile genes in Cryptomeria japonica D. Don with the aid of closely linked markers. Tree Genetics and Genomes 13:61. https://doi.org/10.1007/s11295-017-1149-5

Moriguchi Y, Uchiyama K, Ueno S, Ujino-Ihara T, Matsumoto A, Iwai J, Miyajima D, Saito M, Tsumura Y (2016) A high-density linkage map with 2560 markers and its application for the localization of the male-sterile genes $m s 3$ and ms4 in Cryptomeria japonica D. Don. Tree Genetics and Genomes 12:57. https://doi.org/10.1007/s11295-016-1011-1

Moriguchi Y, Ujino-Ihara T, Uchiyama K, Futamura N, Saito M, Ueno S, Matsumoto A, Tani N, Taira H, Sninohara K, Tsumura Y (2012) The construction of a high-density linkage map for identifying SNP markers that are tightly linked to a nuclear-recessive major gene for male sterility in Cryptomeria japonica D. Don. BMC Genomics 13:95. https://doi.org/10.1186/1471-2164-13-95

Queller DC, Goodnight KF (1989) Estimating relatedness using molecular markers. International Journal of Organic Evolution 43:258-275. https://doi.org/10.1111/j.1558-5646.1989.tb04226.x

Saito M (2010) Breeding strategy for the pollinosis preventive cultivars of Cryptomeria japonica D. Don. Journal of the Japanese Forestry Society 92:316323 (in Japanese with English summary). https://doi.org/10.4005/jifs.92.316 Taira H (2004) Utilization and prospect of Cryptomeria japonica male sterility. Forest Tree Breeding 213:8-11 (in Japanese with English summary).

Taira H, Saitou M, Furuta Y (1999) Inheritance of the trait of male sterility in Cryp tomeria japonica. Journal of Forest Research 4:271-273. https://doi.org/10.1007/BF02762782

Taira H, Saito M, Igarashi M, Saito H (2005) Selection of male-sterile individuals in Cryptomeria japonica. Forest Tree Breeding 216:17-18 (in Japanese).

Taira H, Teranishi H, Kenda Y (1993) A case study of male sterility in sugi (Cryptomeria japonica). Journal of the Japanese Forestry Society 75:377-379 (in Japanese with English summary). https://doi.org/10.11519/jjfs1953.75.4 377

Tsumura Y, Yoshimura K, Tomaru N, Ohba K (1995) Molecular phylogeny of conifers using RFLP analysis of PCR-amplified specific chloroplast genes. Theoretical and Applied Genetics 91:1222-1236. https://doi.org/10.1007/BF00220933

Uchiyama K, Matsumoto A (2018) Development and utilization of a SNP pane for identification of the geographic origin of Cryptomeria japonica. Bulletin of FFPRI 17: 141-148.

Uchiyama K, Miyamoto N, Takahashi M, Watanabe A, Tsumura Y (2014) Population genetic structure and the effect of historical human activity on the genetic variability of Cryptomeria japonica core collection, in Japan. Tree Genetics and Genomes 10:1257-1270. https://doi.org/10.1007/s11295-014-0758-5

Ueuma H, Yoshii E, Hosoo Y, Taira H (2009) Cytological study of a male-sterile Cryptomeria japonica that does not release microspores from tetrads. Journal of Forest Research 14:123-126. https://doi.org/10.1007/s10310-009-0114-z

Wang J (2011) COANCESTRY: a program for simulating, estimating and analysing relatedness and inbreeding coefficients. Molecular Ecology Resources 11:141-145.https://doi.org/10.1111/j.1755-0998.2010.02885.x 
Weir BS, Anderson AD, Hepler AB (2006) Genetic relatedness analysis: modern data and new challenges. Nature Reviews Genetics 10:771-780. https://doi.org/10.1038/nrg1960

Yoshii E, Taira H (2007) Cytological and genetical studies on male sterile sugi (Cryptomeria japonica D. Don), Shindai 1 and Shindai 5. Journal of the Japanese Forestry Society 89:26-30 (in Japanese with English summary). https://doi.org/10.4005/jifs.89.26 